\title{
ALCUNE NOTE SULL'EDILIZIA CIVILE A CASTEL DI CAGLIARI FRA TRECENTO E QUATTROCENTO'
}

\author{
MARIA BONARIA URBAN \\ Università di Cagliari
}

Gli studi su Castel di Cagliari, nonostante gli utili spunti contenuti in alcuni articoli di Giovanni Todde e di Francesca Bocchi ${ }^{3}$, hanno per lo più trascurato di indagare i caratteri dell'edilizia cittadina ${ }^{4}$. Questa imposta-

\footnotetext{
'Per motivi di comodità si utilizzeranno le seguenti abbreviazioni: ACA (Archivo de la Corona de Aragón), ACC (Archivio Comunale di Cagliari), ANTCa (Atti dei Notai della Tappa di Cagliari), ANS (Atti dei Notai Sciolti), ASC (Archivio di Stato di Cagliari), Canc (Cancillería)

'G. TODDE, Disposizioni legislative sull'edilizia a Cagliari nel secolo XIV. in "XIII Congresso di Storia dell'Architettura” (Cagliari 1963), Roma, 1966, pp. 253-260 e IDEM, Strutture abitative a Cagliari dal Quattrocento al Seicento, in La famiglia e la vita quotidiana in Europa dal '400 al '600, "Atti del Convegno (Milano 1983)", Roma, 1986. pp. 447-454.

${ }^{3} \mathrm{~F}$. BосCHI, Regolamenti urbanistici, spazi pubblici, disposizioni antinquinamento e per l'igiene delle maggiori città della Sardegna Medievale, in La Corona d'Aragona in Italia (secc. XIII-XVIII). "XIV Congresso di Storia della Corona d'Aragona (Sassari-Alghero 1990)" (d'ora in avanti CSCA), II, 1, Sassari, 1995, pp. 73-124.

${ }^{4}$ Cfr. D. SCAno, Forma Kalaris. Cagliari, 1934; I. PrInCIPE, Cagliari. Le cittì nella storia d'Italia, Bari, 1981; A. Cossu. Storia militare di Cagliari. 1217-1866. Anatomia di una piazzaforte di prim'ordine 1217-1993. Cagliari, 1993; Cagliari. Quartieri storici, Castello. Cagliari, 1985; Cagliari. Quartieri storici, Marina, Cagliari, 1989; Cagliari. Quartieri storici, Villanova, Cagliari, 1991; Cagliari. Quartieri storici, Stampace, Cagliari, 1995. Sulle fortificazioni: S. CASU, A. DESSI', R. TURTAS, Le piazzeforti sarde durante il regno di Ferdinando il Cattolico (1479-1516), in "XIV CSCA", cit, I, 1, 1995, pp. 217-261, e degli stessi autori, Il "Disegno" di Jacopo Palearo Fratino per il sistema fortificato di Cagliari (15631579), in Arte e Cultura del '600 e '700 in Sardegna, "Atti del Convegno Nazionale (CagliariSassari 1983)», Napoli, 1984, pp. 69-106. All'evoluzione urbana di Cagliari sono dedicati nostri studi: M.B. UrBan. Cagliari catalano-aragonese. Topografia e insediamento nei secoli $X I V$ e XV, tesi di Dottorato di Ricerca; EADEM, Da Bonaria a Cagliari: problemi politici e
} 
zione riflette l'interesse prevalente per l'architettura monumentale e le fortificazioni, gli elementi più appariscenti del paesaggio, ma ha il limite di ignorare le abitazioni private che, quantitativamente predominanti, connotano il volto di una città.

Sicuramente lo studio delle case medievali comporta numerose difficoltà, ma le ricerche interdisciplinari condotte negli ultimi anni su numerosi centri storici italiani hanno focalizzato l'attenzione sul loro ruolo nell'elaborazione dello spazio urbano, per cui diventa indispensabile dedicare ad esse attenzione se si vuole approfondire la conoscenza delle realtà cittadine $^{5}$.

Nonostante la scarsità di dati archivistici e i limiti di una lettura sul campo, una serie di documenti offre spunti significativi per proporre il tema in ambito cagliaritano. Attraverso l'analisi di queste fonti è possibile cogliere alcune caratteristiche delle tipologie costruttive, dei materiali e dei costi delle opere, fornendo al contempo dati interessanti per lo studio dei committenti e delle manovalanze operanti nel corso del XV secolo ${ }^{6}$.

Come è stato già segnalato, Castel di Cagliari aveva raggiunto agli inizi del Trecento una piena maturità’; il primo periodo del governo aragonese venne a coincidere con una nuova fase di sviluppo, però gli strumenti legislativi, in particolare le ordinazioni dei consiglieri cittadini, furono insufficienti per regolare questo processo, tanto che i sovrani aragonesi dovettero intervenire personalmente con provvedimenti ad hoc. I casi di lite fra vicini in materia di pareti comuni, aperture di finestre, scoli, etc., costituivano un problema se nel 1353 Pietro IV, richiamandosi alle

scelte urbanistiche nel primo periodo del regno di Sardegna catalano-aragonese, in «Medioevo. Saggi e Rassegne», 22 (1998), pp. 93-148; EADEM, Nuovi elementi di storia urbana nel regno di Sardegna: dalla fondazione di Bonaria al popolamento catalano di Castel di Cagliari, in "Anuario de Estudios Medievales", 27/2 (1997), pp. 819-866; EADEM, Concessioni in enfiteusi a Castel di Cagliari nel XV secolo, in El poder real en la Corona de Aragón (siglos XIV-XVI), "XV Congreso de Historia de la Corona de Aragón (Jaca 1993)" (d'ora in avanti CHCA), I, Jaca, 1996, pp. 225-254.

${ }^{5} \mathrm{Cfr}$. il volume Case medievali, a cura di Elisabetta De Minicis, che raccoglie le relazioni del Convegno "La città e le case" ed è stato pubblicato nella collana "Storia della città", 52. 1990.

${ }^{6}$ Per motivi di spazio non potremo affrontare l'argomento in modo completo, ci limiteremo pertanto a segnalare alcuni elementi della nostra ricerca, rimandando ad un'altra occasione una sua sistematica trattazione.

${ }^{7}$ G. TODDE, Strutture abitative, cit, p. 449; B. FoIs, Società, struttura urbana, mercati e prodotti alimentari nella Cagliari aragonese del '300, in «Medioevo. Saggi e Rassegne», 15 (1991), pp. 85-108. 
norme esistenti, confermava il divieto di innalzare pareti così come l'apertura di finestre in mancanza di un accordo scritto con i vicini, ma lasciava comunque aperta la strada a nuove costruzioni nel caso di assenza di conflitti ${ }^{8}$.

Le difficoltà politico-economiche sorte ben presto e i danni provocati dalla peste posero un freno ad una crescita ulteriore della città, favorendo piuttosto una sostanziale continuità della forma urbis di impianto pisano per cui, da un confronto con i molteplici esempi di case medievali ancora esistenti a $\mathrm{Pisa}^{9}$, possiamo presupporre che l'abitazione tipica della capitale sarda non doveva essere difforme dal modello studiato dal Lupi e così sintetizzato: «Due pilastri, più spesso tre, raramente quattro, più raramente in numero maggiore, rivestiti di solide pietre quadrangolari o disposte a filari di diversa misura, ossia a struttura pseudoisodoma, erano tirati su all'altezza di circa 10,15 e quasi 20 metri. Le estremità loro erano collegate da archi rotondi od ogivali, secondo lo stile del tempo, della stessa pietra. Il vuoto interno, che ne risultava, era diviso nella sua lunghezza da uno o più architravi di pietra e più tardi da archi scemi fatti di grossi mattoni. Architravi o archi segnavano la linea dei piani, e il vuoto fra un solaio e l'altro non era chiuso in generale da parete, ma spesso da terrazzi sporgenti, che si chiamavano ballatoi. Fra arco ed arco e un poco al di sopra si pareggiava orizzontalmente il muro con pietre uguali a quelle dei pilastri o vi si faceva riposare la parte anteriore del tetto, ovvero il fabbricato si alzava ancora di più per farvi sopra una terrazza coperta o un altro piano o due con aperture più o meno eleganti in facciata e segnatamente con una bifora sopra ogni arco... I fianchi di queste case sono spesso a muro continuo di pietre più piccole e di taglio meno regolare, con finestre disposte senza simmetria, assai piccole e terminate da un architrave a triangolo, posante su stipiti formati di pietre simili a quelle dei pilastri... Molti poi di questi fianchi, specialmente a una certa altezza da terra, erano a muro continuo di mattoni.

${ }^{8} \mathrm{~F}$. BосCHI, Regolamenti, cit, pp. 84-85.

'Sull'edilizia civile pisana esistono vari studi, a partire dalle ricerche ottocentesche di G. ROHAULT DE FleUry. La Toscane au moyen Age. Lettres sur l'architecture civile et militaire en 1400, Paris, 1874 e dal basilare contributo di C. LuPI, La casa pisana e i suoi annessi nel Medioevo, in "Archivio Storico Italiano", XXVII/V (1901), pp. 264-314 e XXVIII/V (1901), pp. 65-96; XXIX/V (1902), pp. 193-227 e XXXII/V (1903), pp. 365-396. L. PERA, L'architettura civile nel periodo medievale a Pisa, s.d. Recentemente si sono occupati dell'argomento F. REDI, Pisa com'era: archeologia, urbanistica e strutture materiali (secc. VXIV), Napoli, 1991 e G. Garzella, Pisa com'era: topografia e insediamento. Dall'impianto tardoantico alla città murata del secolo XII, Napoli, 1990. 
Ma non mancano fiancate con aperture simili a quelle di facciata» ${ }^{11}$. Nel caso cagliaritano si trattava di abitazioni aventi spesso almeno un piano elevato, disposte lungo il fronte stradale e sviluppate nel senso della profondità, con una facciata stretta, al massimo comprendente due luci, e il tetto a spiovente. Il fenomeno della notevole altezza raggiunta dalle case doveva essere rilevante se, subito dopo la conquista catalana, si provvedette alla chiusura del lato interno delle torri" ${ }^{\prime \prime}$.

Sotto la spinta della crescita della popolazione cittadina, la necessità di recuperare nuovi spazi abitativi favorì la diffusione di soluzioni particolari, come quello di costruire a ridosso della cinta muraria; in questi casi la salvaguardia del sistema difensivo e l'esigenza di migliori condizioni abitative trovarono un punto di equilibrio. Ricordiamo un solo esempio: nel 1334 Alfonso il Benigno concesse a Clemen de Salavert, proprietario di una casa contigua al muro regio, di caricare sulla cortina «per fabricari ac fabricare facere et etiam operari et in ipso muro et dicto hospicio vestro construhi facere quandam turrim pro ampliando dicto hospicio»' ${ }^{2}$.

Nel paesaggio urbano caratterizzato da edifici elevati, doveva comunque distinguersi un tipo di costruzione dall' aspetto turrito, riconducibile al modello pisano definito casa-torre ${ }^{13}$. L'imprecisione delle fonti medievali nella definizione di questa tipologia è facilmente spiegabile se si tiene conto che la notevole altezza e una variabile robustezza davano alla dimora civile l'aspetto di una struttura difensiva. Il rapporto tra le due principali dimensioni variò col tempo, le prime sperimentazioni pisane appaiono più vicine a vere e proprie torri, col tempo le forme furono ingentilite da proporzioni diverse e arricchite da decorazioni esterne tipiche delle domus, dando vita a quelle strutture note col nome di torri vergate ${ }^{14}$.

\footnotetext{
${ }^{10} \mathrm{C}$. LuPI, La casa pisana, cit, XXVII/V, pp. 267-268.

"A. Arribas Palau, La conquista de Cerdeña por Jaime II de Aragón, Barcelona, 1952. p. 448 , doc. LVIII.

${ }^{12}$ ACA, Canc, reg. 518 , f. 135, n. 1. Abbiamo analizzato i problemi relativi all'edificazione di dimore private aderenti alle mura nel nostro M.B. URBAN, Cagliari catalano-aragonese, cit.

${ }^{13}$ Il Lupi ritiene che le case-torri siano degli edifici di forma speciale, infatti «dal lato anteriore sono costruiti a tre pilastri come le altre case, salvo che i pilastri sono più bassi e sopra gli archi di congiunzione si eleva il muro continuo per un'altezza assai maggiore che nelle case ordinarien: C. LUPI, La casa pisana. cit, XXVII/V, p. 270. Su questo tema si rimanda all'analisi compiuta da F. REDI, Pisa com'era, cit, pp. 263-ss.

${ }^{14}$ Cfr. F. REDI, Pisa com'era, cit, pp. 254-ss. Lo studioso ha rilevato che le case-torri, inizialmente costruite di pietra, sono successivamente attestate anche in laterizio. Betto Alliata possedeva a Pisa una torre vergata: F. ARTIZZU, Documenti inediti relativi ai rapporti
} 
Nonostante l'assenza di esempi eccezionali come quelli ancora visibili a Pisa, si può comunque affermare che il fenomeno nella sostanza fu presente anche a Castel di Cagliari. Alcuni documenti ricordano che esistevano in città domos sive turres, espressione con cui si era soliti indicare proprio la tipologia della casa-torre ${ }^{15}$. Così sono chiamati due immobili attigui, in muratura e a più piani ubicati nella via dei marinai; costituivano una piccola parte dei beni di Betto Alliata ${ }^{16}$ e si trovavano ubicati nel cuore della città, il terreno su cui sorgevano "tenet unum caput in ruga inferiori Marinariorum et aliud caput in ruga superiori Marinariorum... et aliud latus in platea comunis Castelli Castri», cioè nel tratto centrale della via dei marinai confinante con la piazza comunale ${ }^{17}$.

Le fonti forniscono ben pochi dati sulla qualità dei materiali o sulle tecniche costruttive, ma uno studio comparativo con la realtà pisana ci permette di ampliare le nostre conoscenze. Si può infatti rilevare che gli edifici erano realizzati prevalentemente in muratura, ossia in mattoni o con la pietra estratta dalle cave alle falde del castello. Questa tendenza si diffuse sempre più e nel Trecento divenne predominante, anche se gli interni, le divisioni dei piani e le scale continuarono ad essere costruiti in legno ${ }^{18}$. Purtroppo neanche il censimento degli immobili stilato dai Catalani all'indomani della conquista del regno di Sardegna ci fornisce molte precisazioni a riguardo ${ }^{19}$. Una prima disamina degli elementi urbanistici contenuti nel testo è stata compiuta, ciò ha permesso di individuare, tra l'altro, la gerarchia di valore fra le vie cittadine e la qualità dell'edilizia

economici tra la Sardegna e Pisa nel Medioevo, II, Padova, 1962, p. 125, doc. 59.

${ }^{15}$ F. Artizzu, Documenti inediti, cit, pp. 129-ss, doc. 61.

"Sulle vicende della famiglia Alliata in Sardegna M. TANGHERONI, Una famiglia pisana, gli Alliata, Padova, 1969.

${ }^{17}$ Sulle vicende di queste strutture in epoca aragonese si rimanda al nostro M.B. URBAN, Simboli e strutture del potere: il palazzo regio di Cagliari, in "Quaderni Bolotanesi», 24 (1998), pp. 217-231. Le case-torri dell'Alliata non erano le uniche: Ugolino Pungilupo possedeva una uurris dinanzi alla stessa piazza. Nell'apotheca sita al piano inferiore venne stipulato un contratto riguardante la vendita di un servo: F. ARTIZZU, Docimmenti inediti, cit, p. 17, doc. 12.

${ }^{18}$ Cfr. C. LUPI, La casa pisana, cit, XXVII/V, in part. pp. 283, 294 e 305; F. REDI, Pisa com'era, cit, p. 271.

${ }^{19} R$. CONde I Delgado De Molina, Castell de Cäller. Cagliari catalano-aragonese, Cagliari, 1984. 
cagliaritana ${ }^{20}$; però numerosi dubbi attendono ancora una risposta, perché questa fonte è estremamente avara di elementi utili per identificare le caratteristiche delle abitazioni. Un esempio: nei testi pisani compaiono essenzialmente due vocaboli per definire gli immobili e cioè, domus e hospicium, ma quali sono le differenze fra le due tipologie? Nel censimento si citano i termini casa e alberch, che dovrebbero tradurre i vocaboli precedenti. Le case-torri dell'Alliata sono definite alberchs nel testo catalano, di conseguenza o tutti gli alberchs (e costituiscono in valore assoluto la maggioranza degli immobili censiti) erano delle case-torri oppure bisogna ammettere l'esistenza del problema di interpretare in modo corretto la terminologia utilizzata nelle fonti. Inoltre solo raramente nei documenti si citano le parti strutturali delle case; in un documento del 1463, un edificio ubicato nella via dei mercanti è definito "hospicium sive domus" e viene descritto nel modo seguente: «de abissu fundamentorum dicti hospici ... cum introitibus et exitibus suis ... et cum solis, areis, parietibus, superficiebus et suprapositis, sugrundis, menianis, protectis, stillicidiis et fenestris, portico, botigia et ianuis... $\gg^{21}$. Possiamo stabilire che si trattava di una struttura elevata, probabilmente col tetto a spiovente, dotata di un sistema di scolo delle acque piovane, vari ingressi, una bottega e un portico. Nel $1478 \mathrm{si}$ presentava in modo simile una proprietà ubicata nel vico d'En Bellit, e cioè «quandam domum ... cum duabus botigiis eidem contiguis ... cum introitibus et exitibus, solis, tectis, parietibus et fundamentis» ${ }^{22}$.

In alcuni casi lo scrivano ha fornito un resoconto più preciso che ci aiuta a capire come il nostro tentativo di dividere gli immobili in categorie precise può essere vano a causa delle continue manomissioni a cui erano sottoposti, infatti le unità abitative potevano essere scorporate o ricomposte secondo le esigenze dei proprietari. Nel 1432 i tutori di Angelina Sunyer vendettero un hospicium magnum nella via dei mercanti al catalano Jaume de Linya al prezzo di 1100 lire alfonsine, per estinguere alcune passività maturate dalla pupilla. L'acquisto riguardava non solo la casa, ma anche «quandam quoquinam et quandam retrocameram eidem hospicio magno

\footnotetext{
${ }^{20} \mathrm{Si}$ veda F. BocCHI, Regolamenti, cit, p. 79. La studiosa ricorda che nel censimento sono accatastate 758 unità: 654 alberchs, 55 cases, 31 cases baxes, 7 cases derrocades, 1 endana de tres cases, 1 tinent de cases qui son XI portals, 1 xasso ab VI cases, un certo numero di
} botteghe e un laboratorio.

${ }^{21}$ ASC, Pergamene laiche, n. 28. Su questo documento si veda anche la nota 23.

${ }^{22}$ ASC, ANTCa, ANS, notaio Barbens, 51, prot. 6, ff. 1r-2v (1478 dicembre 28). 
contiguasque edifficate existunt super dicto hospicieto dicto venerabili Simonis Roig, quod existit ad latus dicti magni hospicii versus meridiem, et sunt de pertinenciis dicti hospicieti quequidem quoquina et retrocamera. Volumus comprehendi sub presenti vendicione dicti magni hospicii necnon nominibus antedictis vendimus vobis eidem venerabili Jacobo de Linya et vestris quodam hospicietum dicte pupille situm et positum retro dicti hospicium magnum et eidem magno hospicio contiguum, et habet exitum et introhitum in vico comuniter vocato lo carrer dels mariners et est departimentis dicti magni hospicii cum quibusdam gorgats ibidem constructis. Item eciam nominibus quibus supra vendimus et ex causa vendicionis concedimus vobis eidem Jacobo de Linya et vestris quendam ortetum situm et positum retro dictum magnum hospicium et eidem magno hospicio contiguum, quasi versus dictum vicum marineriorum parietibus circumclausum cum arboribus diversorum generum arboratum, qui ortetus antiquitus erat duo patua duorum hospiciorum ${ }^{23}$. Si trattava insomma di un'ampia e variegata proprietà che, a partire da una struttura autonoma di dimensioni notevoli (hospicium magnum), si era accresciuta con l'annessione di altri ambienti, alcuni dei quali erano in origine inseriti in unità edilizie indipendenti, come la cucina e la "retrocamera" ubicate al piano superiore di un hospicietum contiguo, divenute parte integrante dei beni di Angelina Sunyer. La trattativa includeva anche un piccolo edificio collocato sul lato posteriore del hospicium magnum, fornito di alcuni gorgats, e un orto murato. Tra le caratteristiche fondamentali di questo blocco immobiliare eterogeneo ci sembra rilevante segnalare che, in virtú delle annessioni, si era venuta a creare una proprietà sviluppata nel senso della profondità in modo tale che risultava tangente a due strade e quindi disponeva di più ingressi, esattamente come viene confermato in vari casi simili nel censimento catalano ${ }^{24}$. L'interesse dei proprietari di assicurarsi una maggiore compattezza dei beni ${ }^{25}$, attraverso

\footnotetext{
${ }^{23}$ ASC, Pergamene laiche, n. 10 (1432 marzo 14). Si noti come l'ortetus era in realtà il risultato dell'accorpamento di due più antichi patua. La descrizione delle proprietà, nonostante i nomi dei vicini siano diversi -e ciò potrebbe spiegarsi visto lo scarto cronologico- assomiglia in modo sorprendente a quella contenuta in un'altra pergamena più tarda (ASC. Pergamene laiche, n. 28, 1463 aprile 13), si trattava forse degli stessi beni?

${ }^{2-}$ M.B. Urban, Cagliari catalano-aragonese, cit.

${ }^{25}$ Sin dal 1332 Alfonso il Benigno, modificando uno dei cardini del progetto di popolamento di Castel di Cagliari, concesse il diritto di proprietà di due immobili e quello di abbattere le pareti divisorie per ampliare la superficie a disposizione: M. PINNA, Indice dei documenti cagliaritani del Regio Archivio di Stato dal 1323 al 1720, Cagliari, 1903, p. 22, n. 90.
} 
l'abbattimento delle pareti divisorie, produceva come conseguenza piú importante l'estensione della superficie abitabile in senso orizzontale, e ciò costituisce un elemento di profonda novità rispetto alle più antiche cellule abitative di impianto pisano, generalmente disposte su più piani, ma estremamente ridotte nel senso dell'ampiezza ${ }^{26}$.

Se il tessuto urbano di Castel di Cagliari si mantenne sostanzialmente inalterato nel corso del Trecento, fu il rovinoso incendio del 7 agosto del $1386^{27}$, che distrusse più di un centinaio di case fra le migliori, a creare le condizioni per un massiccio rinnovamento dell'assetto edilizio. Una parte delle distruzioni in realtà, furono provocate per fermare l'avanzata delle fiamme; due dei tre edifici ubicati nella via dei mercanti appartenenti ad Arnau Çoroll per esempio, furono parzialmente abbattuti «pro salvacione aliorum hospiciorum dicti Castri ${ }^{28}$. Per far fronte a questo disastro, che aveva intaccato il patrimonio edilizio e provocato gravi danni al sistema economico della città, la corte non progettò alcuna riforma complessiva dell'assetto urbano, dal momento che i re Giovanni e Martino si limitarono ad ordinare l'annullamento dei censi gravanti sugli immobili abbattuti e anche l'applicazione di tale provvedimento dovette essere gravemente contrastata, se si tiene conto che venne più volte rinnovato e lo stesso Arnau Çoroll decise di recarsi personalmente a Barcellona per esporre la sua dolorosa situazione ${ }^{29}$.

Nonostante la mancanza di un piano di ricostruzione, fu l'iniziativa privata a tentare di porre rimedio ai danni provocati dal disastro. Finora abbiamo raccolto solo sporadiche testimonianze, però ci sembrano interessanti perché pongono in evidenza la libertà, $o$ forse sarebbe meglio dire l'illegalità, con cui si agì. Una prova di ciò si trova in due lettere di Martino il Vecchio indirizzate ai principali ufficiali del regno e al consiglio cittadino,

\footnotetext{
${ }^{26} \mathrm{Cfr}$. F. Redi, Pisa com'era, cit, pp. 186-ss.

${ }^{27}$ F.C CASUla, Carte reali diplomatiche di Giovanni I il Cacciatore, re d'Aragona, riguardanti l'Italia, Padova, 1977, pp. 33-34, docc. 1-2; pp. 94-95, doc. 71. ASC, AAR, B5, f. 263r-v. Un riscontro preciso sulla data dell'incendio abbiamo trovato in ACA, Canc, reg. 2209, f. 5r-v e ff. 14v-15r (il primo dei due documenti non fu spedito).

${ }^{28}$ F.C. Casula, Carte reali, cit, p. 33. Lo studioso ricorda che il Coroll pagava per due edifici un censo annuale di 30 lire a favore della chiesa di Santa Maria di Castel di Cagliari, per il terzo un censo di 20 lire alla chiesa di San Giacomo di Villanova.

${ }^{29} A C A$, Canc, reg. 2226, ff. 14v-15r; f. 38v; f. 63r-v; ff. 63v-64v; ff. 110-111r; ff. 112v$113 r$. In seguito all'incendio, i consiglieri di Castel di Cagliari presero una serie di provvedimenti per limitare il rischio di nuovi incendi, tra cui la proibizione di costruire ballatoi lignei: G. TODDE, Strutture abitative, cit, p. 450.
} 
in cui il sovrano ordinava di non perseguire Jaume Xarch e Ramon Boter per il modo in cui avevano provveduto alla ricostruzione delle loro case ${ }^{30}$. I nuovi edifici erano stati realizzati di dimensioni maggiori di quelle originarie, sottraendo porzioni di suolo pubblico. Martino ordinava di non intentare alcuna azione nei confronti dei due proprietari, nonostante nella «fabricacione memorati hospicii ... fundamenta, parietes vel alia edificamenta seu constructiones eiusdem hospicii ultra priscos vel solitos terminos seu limites extendi vel etiam evagari et propterea quitquam a via regia seu publica occupasse ultra limites solitos et antiquos dictum hospicium extensum seu elongatum extiterit» ${ }^{31}$. Questo esempio dimostra come, nonostante la legislazione esistente, si verificassero casi di abuso edilizio, talvolta tollerati dallo stesso sovrano, se si trattava di beneficiare i fedeli servitori della Corona $^{32}$.

La situazione critica in cui versava il regno sardo alla fine del Trecento fu sicuramente una delle ragioni principali del mancato avvio di un'opera di riforma urbana e il forte calo demografico registrato in città contribuiva ad aggravare la situazione ${ }^{33}$. Quando si dovevano compiere interventi di restauro delle abitazioni e le condizioni economiche non permettevano un'immediata disponibilità di denaro, gli abitanti potevano ricorrere alla richiesta di un prestito da saldare con l'istituzione di un censo gravante sulla proprietà immobiliare a favore dei creditori ${ }^{34}$. Questa strada

\footnotetext{
${ }^{30}$ ACA, Canc, reg. 2226, f. $174 r-v$ e ff. $174 v-175 r$.

${ }^{31} \mathrm{ACA}$, Canc, reg. 2226, ff. $174 \mathrm{v}-175 \mathrm{r}$.

${ }^{32}$ Un altro esempio: nel 1452 Alfonso il Magnanimo concesse a Joan Soclam la licenza di costruire quandam scalam in prossimità di alcune abitazioni della piazza comunale, con la facoltà di decidere l'ampiezza e l'esatta ubicazione (ACA, Canc, reg. 2636, ff. 127r-128r).

${ }^{33}$ Sulla situazione demografica mi limito a ricordare gli studi di C. LIVı, La popolazione della Sardegna nel periodo aragonese, in "Archivio Storico Sardo", XXXIV, 1984, pp. 23-130 e J. DAY. Uomini e terre nella Sardegna coloniale: XII-XVII secolo. Torino. 1987.

${ }^{34}$ M.B. URBan, Cagliari catalano-aragonese, cit. Talvolta si cedeva un censo di cui si beneficiava in cambio di una somma in contanti, come nel caso dei coniugi Rafael e Caterina Gener e Joana, moglie di Blai Pellicer, che vendettero un censo di tre lire alfonsine a Joan Cerver, per procurarsi il denaro utile alla riparazione della loro casa nel carrer dels mariners, confinante ad occidente con la casa del pittore Joan Figuera (1463 ottobre 13): in Culturc quattro-cinquecentesca in Sardegna. Retabli restaurati e documenti, Cagliari, 1984, p. 167, n.
} 
continuò ad essere seguita per tutto il XV secolo, generando una moltiplicazione del sistema censuale ${ }^{35}$.

La difficoltà di studiare il tessuto edilizio si accresce, se tentiamo di affrontare il tema relativamente allo sviluppo delle appendici. Al momento della conquista catalana le tre ville non avevano registrato la stessa forte crescita del castrum, ma le attestazioni di domus ballatoriatae e la preesistenza di strutture porticate sono prove inconfutabili che anche qui l'edilizia rispondeva ai canoni cittadini ${ }^{36}$. Soltanto nel corso del XV secolo, quando si accesero i primi segnali di una lenta ripresa economica, si verificò un forte aumento del numero di concessioni in enfiteusi di terreni da edifica$\mathrm{re}^{37}$, in questo modo la corte riversò sui privati l'obbligo di costruzione o miglioria dei terreni. L'evoluzione del tessuto edilizio in Stampace costituisce a proposito un caso emblematico. Il 29 agosto 1414 Gantino de Bosa ottenne un terreno «satis prope ipsam ecclesiam de dicta Sancta Restituida" per la costruzione di un portico; il lotto, delle dimensioni pari a 4 canne e mezza di longitudine e 1 canna e 7 palmi di latitudine, era confinante a oriente con l'abside della chiesa e a nord con un'altra struttura porticata di proprietà di Bernat de Bonahora. Poco tempo dopo, nel marzo 1415 , il miles Joan de Sena ricevette un pezzo di terreno prope portale ipsius ville e confinante con la piazza, con alcune vie e il tenedonis di Joan

\footnotetext{
${ }^{35} \mathrm{Nel} 1456$ i coniugi Joan e Isabel Genis comprarono una casa appartenente alla vedova di Joan Maguerola, confinante da un lato con il muro regio, dagli altri con le proprietà di Francesc Siurana e di Lluis Çafranquesa. Due anni dopo la coppia chiese a Joan e Isabel Rassit un prestito di ottocento lire, che venne saldato attraverso l'istituzione di censo annuale a favore dei creditori: ASC, ANTCa, ANS, notaio Garau, 337, prot. 2, ff. 164v-ss; ASC, ANTCa, ANS, notaio Daranda, 254 , prot. 2 , f. $5 \mathrm{r}$ e prot. 4 , ff. $23 \mathrm{r}-24 \mathrm{v}$. Fecero altrettanto nel 1482 i coniugi Simon e Juliàna Vaccarella per compiere i lavori di ricostruzione della loro casa in Villanova "que minatur ruhinam»: ASC, ANTCa, ANS, notaio Barbens, prot. 12, II, ff. $8 \mathrm{r}-11 \mathrm{r}$.

${ }^{36} \mathrm{ASC}$, ANTCa, ANS, notaio Garau, 337, prot. 1, f. 12r e f. 29r-v. ASC, ANTCa, ANS, notaio Barbens, 51, prot. 4, ff. $11 \mathrm{r}-12 \mathrm{v}$. ACA, Canc, reg. 2209, f. $24 \mathrm{r}-\mathrm{v}$ : questa fonte ricorda tres staticas sive contiguas domos cum tribus portalibus ubicate in Villanova. Esempi di domus ballatoriatae nelle appendici sono attestate nei documenti editi da F. ARTIZZU, Documenti inediti, cit, pp. 131-ss, doc. 61 .

${ }^{37}$ Sulle concessioni in enfiteusi risalenti al XV secolo, che si inserirono in un progetto più generale di riforma dell'amministrazione del regno sardo: M.B. URBAN, Concessioni in enfiteusi, cit. Sul sistema enfiteutico e le conseguenze della sua diffusione nella crescita di nuovi borghi nel territorio di Barcellona si vedano le ricerche di J.R. JULIA VIÑAMATA, La enfiteusis como factor transformador del paisaje en la Barcelona de siglo XIV, in "Universitas Tarraconensis", X (1991), pp. 129-149, e di M. RIU RIU, La financiación de la vivienda, propriedad horizontal y pisos de alquiler en la Barcelona del siglo XIV, in La ciudad hispanica durante los siglos XIII al XVI, II, Madrid, 1985, pp. 1397-1405, da cui è possibile ricavare tutta la bibliografia sull'argomento.
} 
Sorroch, con l'obbligo di realizzare un'abitazione ed un portico ${ }^{38}$. Le dimensioni del lotto risultavano pari a 11 canne e 2 palmi, di cui 9 canne e 2 palmi avrebbero costituito la superficie della casa, mentre le restanti due sarebbero state utilizzate per il porticato. Nello stesso anno una concessione molto simile fu firmata a favore di Miquel Canoll, carnisser abitante della medesima villa. Questi otteneva nella via Santa Margherita un pati di 8 canne di longitudine e 3 canne e mezzo di latitudine, l'appezzamento risultava confinante con la roccia del castrum, con un altro terreno dello stesso beneficiario e parte, infine, con la piazza ${ }^{39}$.

Sicuramente il volto della città e delle appendici si modificò incessantemente nel corso del XV secolo soprattutto in virtù dei continui lavori di restauro o manutenzione degli immobili esistenti. Nel 1454 per esempio, per dirimere una controversia sorta tra Madalena de Carmona e Antoni Masa, abitanti di Stampace, gli obrers della città, sulla base del giudizio espresso dai maestri muratori Antoni Barrai, Gerard Carroch e Antoni Morroni, ordinarono alla donna di «alçiare parietem a parte retro eius domus, furni existente per duos palmos supra parietem domus dicti Anthoni Masa». L'opera avrebbe sanato la vertenza fra i due vicini attraverso la chiusura de «los forats que són en la paret a migera entre lo dit Anthoni e la dita casa del forn per forma que non pas lo fum» ${ }^{40}$. L'anno seguente, Pere Lobera si impegnò ad ampliare una casa situata presso l'ospedale di Sant'Antonio nella Lapola, sopraelevando un vano con finestra, dove i due pittori barcellonesi Rafael Tomàs e Joan Figuera avrebbero potuto lavorare alla realizzazione del retablo di San Bernardino destinato alla chiesa di San Francesco ${ }^{41}$.

Al di là dei dati segnalati, mancano quasi del tutto gli elementi per individuare i tipi di restauro compiuti, a questo proposito ci sembra molto significativo un atto notarile sardo che si riferisce alle modifiche apportate ad un edificio cittadino. Vediamo nei dettagli il suo contenuto.

\footnotetext{
${ }^{38}$ ASC, AAR, BD1, ff. $3 r-4 r$ e ff. $14 v-16 v$.

${ }^{39} \mathrm{ASC}, \mathrm{AAR}, \mathrm{BD} 1$, ff. $29 \mathrm{r}-30 \mathrm{r}$.

${ }^{40}$ ASC, ANTCa, ANS, notaio Garau, 337, prot. 2, f. 48r.

${ }^{41}$ Cultura quattro-cinquecentesca, cit, p. 165, n. 35; si veda ibidem, n. 36, a proposito degli oggetti che arredavano la casa affittata dai due pittori. Cfr. A. GALLISTRU, Dimore cagliaritane negli atti notarili, in Retabli. Arte sacra in Sardegna nei secoli XV e XVI, Cagliari, 1993, p. 202 .
} 
Il $1^{\circ}$ ottobre del 1456 Ramon Desbanchs vendette una domus al notaio Jaume Cassa; il denaro ricavato dall'affare venne subito dopo investito per realizzare i lavori nella sua abitazione ubicata nel carrer dels pellicers $^{42}$. Il giorno 29 dello stesso mese, Ramon si presentò dal notaio Joan Guerau e si accordò con il mestre de cases Antoni Morroni sulle condizioni del contratto, che era una scarada, cioè a cottimo ${ }^{43}$. Il costo dell'opera era stato fissato ad una cifra di poco superiore alle 380 lire; a inizio dei lavori il Desbanchs avrebbe pagato 126 lire 14 denari 4 soldi, la stessa somma sarebbe stata versata a metà dell'opera e una quantità pressoché identica a conclusione dell'intervento. Il Morroni infine, si impegnava ad iniziare il lavoro non appena avesse concluso quello in corso nell'abitazione d'En Aymerich e avrebbe accettato ulteriori incarichi soltanto con l'approvazione del Desbanchs ${ }^{44}$.

L'opera prevedeva l'occlusione completa della struttura porticata ubicata al pianoterra («hun arc de peu de fonament fins en lo loch hon d'en cloure en sa forma [***] ha amuntar tot lo front de la dita casa sobre l'arc, fins a parapits de la dita cuberta»), sulla facciata si sarebbero collocate una "finestra dobla e una finestra petita cascuna part», dello stesso stile degli infissi dell'abitazione del vicino Andreu Aymerich, e due ingressi, uno per la bottega e l'altro per condurre alla sala ubicata al piano superiore. Il maestro avrebbe unito la nuova parete con il muro laterale attraverso una cortina di congiunzione dal lato dell'abitazione di Joan Raset («brancha de calsina ... fins a legar la paret vella que ara és a la paret closa ab la brancha de calsina» $)^{45}$. Il contratto conteneva una descrizione precisa anche degli aspetti minori del lavoro che, purtroppo, è andata quasi completamente perduta; sappiamo comunque che il proprietario si sarebbe assunto l'onere di procurare il legname per i ponteggi, le tavole, le corde, gli xebrons, ma non avrebbe dovuto fornire l'acqua né gli altri materiali (calce, pietre, etc.).

\footnotetext{
${ }^{42}$ ASC, ANTCa, ANS, notaio Garau. 337, prot. 2, f. 172v e f. 180r-v.

${ }^{43} \mathrm{Cfr}$. V. Porru, Dizionario sardo-italiano, Cagliari, 1866, s.v. scarada.

"ASC, ANTCa, ANS, notaio Garau, 337, prot. 2, f. 180r-v. Il documento si presenta di difficile interpretazione, dal momento che l'inchiostro è svanito per ampi tratti; per quanto riguarda la spesa totale del lavoro, se la nostra lettura è corretta, raggiungeva la cifra di 380 lire 2 soldi.

${ }^{45}$ Cfr. A.M. Alcover, Diccionari catalì, valenciò, balear, II, Palma de Maiorca, 1985, p. 650, s.v. branca; si chiamava così la serie di pietre poste ad angolo per rinforzare due pareti che si congiungevano o quelle collocate a formare la cornice di una porta o finestra. Nel nostro caso il vocabolo sembra riferibile alla prima accezione.
} 
Nonostante le lacune del testo, ci sembra di poter affermare che il tipo di intervento stabilito dal contratto avrebbe modificato nella sostanza l'aspetto dell'edificio. La casa del Desbanchs era in muratura e a più piani, la superficie abitabile sarebbe stata accresciuta dalla chiusura del portico, la cui pertinenza giuridica aveva da sempre suscitato dispute fra i cittadini e l'amministrazione urbana. Una conferma che i lavori prevedessero l'occlusione dell'ambiente aperto al pianoterra ci sembra possa essere riconosciuta nell'esigenza di unire la parete già esistente con la nuova, attraverso la realizzazione di una sezione angolare. Il testo accenna all'apertura di due ingressi sulla facciata costruiti esternamente con l'uso di blocchi squadrati di pedra picada, cioè il calcare estratto dalle cave cittadine, in un pezzame sbozzato e regolare, mentre la parte interna sarebbe stata de coble, termine con cui si indicava una miscela di arena e pietrame di dimensioni ridotte ${ }^{46}$. Il riferimento ad un portalet ligneo sembrerebbe in relazione a una delle due porte d'accesso, ma le indicazioni del documento sono su questo punto molto frammentarie.

Per permettere un'adeguata illuminazione degli ambienti, si sarebbero predisposte alcune finestre sulla facciata. È interessante notare che il Desbanchs chiedesse la realizzazione di un particolare tipo di infissi, poiché si trattava di riprodurre le finestre dell'abitazione di Andreu Aymerich ubicata nella stessa via; inoltre il richiamo alla finestra dobla si riferisce alle eleganti bifore gotiche che andarono a ingentilire i prospetti delle abitazioni cagliaritane nel corso del Tre-Quattrocento, ma di cui purtroppo sopravvivono solo pochissimi resti ${ }^{47}$.

Nel complesso l'intervento avrebbe comportato un profondo rinnovamento e abbellimento della casa. Il pianoterra aperto segnato dalla successione di una o due arcate, caratteristica fondamentale dell'edilizia pisana, doveva essere limitatamente diffuso nella città sarda se si crede ai dati contenuti nel censimento catalano ${ }^{48}$. A questo modello doveva peró

\footnotetext{
${ }^{46}$ Sui materiali nell'edilizia medievale sarda cfr. M. PINNA. Le ordinazioni dei comsiglieri del Castello di Cagliari nel secolo XIV. Cagliari. 1927, p. 46, n. 88, p. 66, n. 128, p. 120. n. 48. p. 148, n. 105; G. SPIGA, Le pietre da taglio nelle fortificazioni medioevali di Sardegna. in "Medioevo. Saggi e Rassegne", 15 (1990). pp. 243-254. 1965.

${ }^{47}$ Per un utile confronto si rimanda allo studio di V. Moss A, Architetture sassaresi, Sassari,

${ }^{48}$ F. BocCHI, Regolamenti urbanistici, cit, p. 82: sulla base degli elementi contenuti nel censimento catalano, la studiosa conta solo trenta occhi, cifra bassa, a suo dire, per una città commerciale, ma piuttosto comprensibile per la prevalente vocazione mercantile di Castel di
} 
corrispondere la casa del Desbanchs che, secondo consuetudine, ospitava la bottega accanto all'ingresso, da cui si accedeva agli ambienti collocati nei livelli superiori ${ }^{49}$.

L'edificio doveva essere di pregio e non costituiva la dimora media, in quanto solo le famiglie benestanti potevano permettersi una casa realizzata nel rispetto di soluzioni stilistiche ricercate, di materiali resistenti e in particolare, di pietre finemente lavorate, ma non era un'eccezione perché alcuni spunti confermano confermare la presenza di casi simili, per esempio il riferimento alle finestre e ai lavori in corso nella casa del vicino Andreu Aymerich avvalora l'ipotesi di un livello edilizio non disprezzabile e soggetto nel corso della seconda metà del Quattrocento a sensibile miglioramento ${ }^{50}$. Inoltre, una ricerca sommaria sulle condizioni economiche e sociali degli abitanti residenti nei pressi della casa del Desbanchs sembra suggerire un'agiatezza diffusa; l'area gravitante attorno al carrer dels pellicers e i tratti inferiori del carrer dels mercarders e del carrer del mariners ospitavano vari notai, un dottore in leggi e soprattutto molti commercianti, si trattava pertanto di un settore con una massiccia presenza di esponenti del ceto medio $^{51}$.

Agli elementi contenuti nel contratto si può aggiungere una considerazione che emerge dal confronto con la documentazione coeva. Il calo demografico verificatosi in città fra Tre e Quattrocento dovette favorire una crescita degli spazi inoccupati in particolare all'interno del castrum, mentre si affermava una tendenza generale ad un uso più razionale degli ambienti, senza ricorrere all'edificazione di nuovi piani, circostanza che viene confermata, per esempio, dal restauro compiuto nella dimora del

Cagliari.

${ }^{49}$ Sul termine sala, voce di origine longobarda, cfr. G.B. PELLEGRINI, Attraverso la toponomastica urbana medievale in Italia, in Topografia urbana e vita cittadina nell'alto medioevo in Occidente, "XXI Settimane del Centro di Studi sull'Alto Medioevo (Spoleto 1973)", tomo II, Spoleto, 1974, p. 427 e F. REDI, Pisa com'era, cit, pp. 303-ss. Il Lupi ricorda che negli edifici a più piani abitati da una sola famiglia si trovavano essenzialmente tre ambienti: la sala, la cucina, la camera da letto; la cucina era in genere collocata nel piano più alto per un facile e sicuro sfogo del fumo: C. LUPI, La casa pisana, cit, XXVII/V, p. 307 e pp. 311-312.

${ }^{50} \mathrm{Cfr}$. ASC, ANTCa, ANS, notaio Barbens, 51, prot. 6, ff. $1 \mathrm{r}-2 \mathrm{v}$ e ibidem, notaio Durante, 268 , prot. 2, f. 123 r-v.

${ }^{51}$ ASC, ANTCa, ANS, notaio Garau, 337, prot. 2, f. 172v; ASC, ANTCa, ANS, notaio Durante, 268, ff. 20r-22r e f. 61v; ASC, ANTCa, ANS, notaio Barbens, 51, prot. 12, f. 50r-v; ASC, AAR, BD15, ff. 13r-14r e f. 60r, nn. 1-2. 
Desbanchs e finalizzato ad una migliore ridistribuzione del volume abitativo $^{52}$.

I dati contenuti nel contratto del 1456 acquistano maggiore rilievo se si tiene conto di un'altra testimonianza dell'attività edilizia a Castel di Cagliari risalente al 1470 , già segnalata dal Todde ${ }^{53}$. In quell'occasione Joan de Mosques, abitante dell'appendice di Villanova, affidò ai magistri domorum Antoni Morroni e Julià Manus il compito di ristrutturare la sua dimora $^{54}$. I due maestri muratori si impegnarono a costruire entro un semestre «a la casa e front del dit Johan de Mosques una arquada e archets de bona pedra fins a la primera cuberta, a compliment del primer entaulament complidament»; ma nel caso che prima dello scadere dei tre anni dalla conclusione dell'opera "la pedra o pedres se venia a menjar» i muratori avrebbero dovuto garantire la sostituzione dei pezzi usurati o mancanti. Il costo dei materiali, comprendenti «pedra e calsina, arena, aygua, cubells, gavetes, palamerio, cordes e tot lo lenyam, axí per pons com per síndries», e il vitto dei manovali sarebbero gravati sui maestri. La spesa prevista per l'intervento, pari a settanta lire, doveva essere ripartita in tre quote, a inizio, a metà e a fine d'opera. In caso di violazione degli accordi venne fissata una multa di 25 lire, da dividersi in parti uguali tra la corte e l'opera della chiesa di S. Domenico.

Facendo un confronto con il contratto precedente, possiamo trovare numerosi ed interessanti elementi in comune. In primo luogo, i due lavori erano caratterizzati da un desiderio di abbellimento della struttura, in particolare della facciata, con l'introduzione di arcate e finestre ispirate alla decorazione gotica. Come nei lavori compiuti nella casa di Ramon Desbanchs inoltre, si cercava di rinnovare i caratteri stilistici dell'edificio attraverso l'introduzione di modelli conformi al gusto prevalente. Un'ulteriore conferma di questa tendenza si trova in un contratto del 1453: i mestres de cases Andreu Joan Falch e il Tamborino avrebbero realizzato nella dimora di Pere Iner due archi bassi, simili a quelli della casa di Mateu Serra,

\footnotetext{
${ }^{52}$ Un documento notarile del 1463 , in cui si parla di una dimora ubicata nel carrer dels pellicers di proprietà di Gabriel Descoll, ci offre a proposito ulteriori spunti di riflessione. Nell'ambito dei capitoli stipulati in occasione delle nozze della figlia Tomasa con il mercante Joan Andreu, il padre della giovane, animato da amor paternal, stabiliva, tra l'altro, di lasciare agli sposi la sala e cambra e soster mes amunt della sua casa per un triennio senza l'onere di pagare l'affitto: ASC, ANTCa, ANS, notaio Durante, 268, prot. 2, ff. 31r-32v.

${ }^{53} \mathrm{G}$. TODDE, Strutture abitative, cit, p. 452.

${ }^{54}$ ASC, ANTCa, ANS, notaio Barbens, 51, prot. 13, ff. 40r-41v (1470 settembre 11).
} 
e una serie di luci al costo di 180 lire. Alla conclusione dei lavori, la facciata sarebbe stata cadenzata, in senso verticale, dalla successione di due archi scemi alla base e di alcune finestre al piano superiore ${ }^{55}$.

Al di là degli indizi costruttivi, i contratti offrono alcuni elementi utili per identificare le manovalanze che realizzarono le opere: uno dei due maestri interpellati da Joan de Mosques era, se non lo stesso Antoni Morroni che aveva restaurato la casa di Ramon Desbanchs, certamente un esponente della medesima famiglia di muratori originari di Stampace ${ }^{56}$. Numerosi elementi confermano che la loro bottega era riuscita a conquistarsi un ruolo di primo piano nel mercato urbano; non a caso Antoni Morroni fu interpellato dal consiglio cittadino, per dare il suo parere di esperto a proposito della lite sorta fra due vicini, e vari componenti della medesima famiglia occuparono un ruolo di rilievo nella gestione della confraternita dei falegnami e dei muratori, sin dal momento della sua costituzione nella seconda metà del Quattrocento ${ }^{57}$.

\section{RÉSUMÉ}

Au début du XIV" siècle Castel di Cagliari avait atteint un notable niveau de développement urbain caractérisé par la diffusion de modèles de construction typiquement italiens. Ce processus de croissance subit un brusque ralentissement à la suite des problèmes engendrés par la conquête catalane; la ville vint à manquer des ressources nécessaires à une

\footnotetext{
${ }^{55}$ ASC, ANTCa, ANS, notaio Daranda, 254. prot. 2, f. 43v. Il documento. regestato in $L a$ Corona d'Aragona: un patrimonio comme per Italia e Spagna (secc. XIV-XV), Cagliari, 1989. p. 420. n. 419, contiene piú elementi di quelli da noi riportati, ma la loro interpretazione suscita molti dubbi: "los dits mestres són tenguts e obligats al dit Pere Ynery fer dos arches baxes en la enfront de la sua casa, obra semblant de la de l’arch d’En Matheu Serra. Item obra plana e de aqui anants lo enfront fins a la segona cuberta ab tres finestres com obra plana e dos archs ab cascú, co és a cascú corp del enfront...". Sul significato di obra plana si rimanda a A.M. ALCOVER, Diccionari catalì, cit, s.v. plana.

${ }^{56}$ ASC, ANTCa, ANS, notaio Garau, 337, prot. 2, f. 180r-v.

${ }^{57}$ Vedi supra, nota 40. Cfr. G. OLLA REPETTO, L'organizzazione del lavoro a Cagliari tra '400 e '500: la confraternita dei falegnami. in Sardegna, Mediterraneo e Atlantico tra Medioevo ed età moderna. Studi storici in memoria di Alberto Boscolo, a cura di L. D'Arienzo). I. Roma, 1993. pp. 429-448. Abbiamo analizzato il ruolo della famiglia Morroni e delle principal botteghe cittadine in: M.B. Urban, Cagliari catalano-aragonese cit. Sulle corporazioni sarde si rimanda a S. LIPPI, Statuti delle corporazioni d'arti e mestieri. Cagliari. 1906; R. DI TUCCI. Le corporazioni artigiane della Sardegna (con statuti inediti), in "Archivio Storico Sardom. XVI (1926), pp. 33-160; F. LODdo CANEPA, Statuti inediti di alcuni gremi sardi, ibidem. XXVII (1961), pp. 177-442.
} 
ultérieure expansion et conserva substantiellement le même aspect jusqu'à la fut du XIV siècle.

Au XV' siècle une lente reprise économique favorisa l'extension des faubourgs et de nombreux travaux de restauration des structures existantes. Cette phase de croissance se distingua par une adéquation des modèles urbains précédants au goût gothique alors dominant.

Une série de documents nous permettent d'éclairer certains aspects de la question et d'identifier les types d'interventions réalisées dans les maisons cagliaritaines du bas MoyenÂge.

\section{SUMMARY}

In the beginning of XIV century Castel di Cagliari had obtained a considerable urbanistic development characterised by the diffusion of building models typically Italian. The growing process had a sudden slowing down according to some problems born after the Catalan conquest. Lost the resources for a further spread, the town maintained substantially its aspect until the end of the fourteenth-century.

In the XV century a slow economic recovery favoured the building spread of the adjoining districts, where we can notice many restoration interventions of the existent structures. This growing period has been characterised by an adaptation of the huilding models, preceding the gothic style.

Several documents permitted to enlighten on some aspect of the subject and to identify the kind of interventions made on the houses of Castel di Cagliari in the Late Middle Age. 